\title{
Photosensitized Oxidation of 9,10-Dimethylanthracene on Dye-Doped Silica Composites
}

\author{
Elim Albiter, Salvador Alfaro, and Miguel A. Valenzuela \\ Laboratorio Catálisis y Materiales, ESIQIE Instituto Politécnico Nacional, Zacatenco, 07738 México City, DF, Mexico \\ Correspondence should be addressed to Miguel A. Valenzuela, mavalenz@ipn.mx
}

Received 15 July 2011; Accepted 17 November 2011

Academic Editor: Shifu Chen

Copyright () 2012 Elim Albiter et al. This is an open access article distributed under the Creative Commons Attribution License, which permits unrestricted use, distribution, and reproduction in any medium, provided the original work is properly cited.

A series of cationic dyes, methylene blue (MB), safranin O (SF), toluidine blue (TB), and neutral red (NR), were successfully incorporated into a silica matrix by using ultrasound irradiation during the Stöber process. Several analyses were performed, including scanning dynamic light scattering (DLS), electron microscopy (SEM), nitrogen physisorption, FTIR spectroscopy, UVvis, and diffuse reflectance spectroscopy. The entrapped dyes on silica were evaluated in singlet oxygen $\left({ }^{1} \mathrm{O}_{2}\right)$ generation under visible light irradiation, by means of the photosensitized oxidation of 9,10-dimethylanthracene (DMA). According to the results, the photocatalytic performance of the silica composites was improved, and the leakage of the dye from the particles was suppressed. Among these four different types of dye-doped silica composites, the $\mathrm{SiO}_{2}-\mathrm{SF}$ composite showed the most efficient delivery of ${ }^{1} \mathrm{O}_{2}$.

\section{Introduction}

A photosensitized reaction is defined as the process leading to photochemical or photophysical changes in a substrate by means of the absorption of radiation by other entity called a photosensitizer [1]. Since the 1960s, several fundamental works have been published related to photosensitization in presence of oxygen and have distinguished two competing mechanisms referred as Type I and Type II [2-5]. Type I photooxygenation involves the formation of a sensitizer triplet state $\left({ }^{3} \mathrm{~S}^{*}\right)$ which interacts with a substrate $(\mathrm{XH})$ giving rise to a pair of free radicals by electron-transfer or hydrogentransfer mechanisms [1]. These produced radicals react with oxygen to regenerate the sensitizer and to form peroxy or superoxide radicals. Type II mechanism implicates the direct interaction of the sensitizer excited state with oxygen, generating upon energy transfer singlet oxygen $\left[{ }^{1} \mathrm{O}_{2},\left({ }^{1} \Delta_{\mathrm{g}}\right)\right]$ which reacts directly with numerous organic substrates [6].

In spite of many efforts and successful results in the study of photosensitized reactions in homogeneous media, there are some disadvantages by this route mainly due to the solubility of the sensitizers in the reaction solvent and their removal from the reaction mixture [7]. These problems can be overcome by the immobilization of the sensitizer in appropriated solid carriers. For instance, the advantages of using a dye dispersed on a solid carrier are the oligomerization of the dye can be prevented, a higher photostability of the dye is obtained, a higher purity of the final products, and the reusability of the dye [8]. Important applications, for example, fine chemical synthesis, wastewater treatment, and photodynamic processes, among others, have been found for photosensitized singlet oxygen production [7-14].

Although many solid carriers such as polymers, zeolites, semiconductors, glasses, silica gel, among others, have been used to support a variety of sensitizers [15], we are interested to develop a hybrid organic-inorganic systems made of dyedoped silica particles active under visible-light irradiation for fine chemical synthesis. Silica is a very attractive material for many industrial and medical applications because it is inexpensive, chemically inert, thermally stable, and biocompatible. In fact, silica particles have captured much attention over the past two decades for their application in catalysis, separation, biosensors, and adsorption [16]. Silica particles can be tuned from 50 to $300 \mathrm{~nm}$ containing pore diameters between 2 and $10 \mathrm{~nm}$ allowing for different dyes loadings. Also, they have a high surface area $\left(>700 \mathrm{~m}^{2} / \mathrm{g}\right)$ and large pore volume $(>0.9 \mathrm{~mL} / \mathrm{g})$ allowing high loading of chemicals [16]. The doping of organic dyes into a silica matrix is not an easy task due to the weak interaction between the organic-inorganic hybrid compound [17]. Usually, the main 
<smiles>Cc1cc2nc3ccc(=[N+](C)C)cc-3[nH]c2cc1N</smiles>

III<smiles>Cc1cc2nc3cc(C)c(N)cc3[n+](-c3ccccc3)c2cc1N</smiles><smiles>CN(C)c1ccc2nc3ccc(=[N+](C)C)cc-3sc2c1</smiles><smiles>Cc1cc2nc3ccc(=[N+](C)C)cc-3sc2cc1N</smiles>

Figure 1: Molecular structure of the dyes: (I) neutral red (NR), (II) methylene blue (MB), (III) safranin O (SF), and (IV) toluidine blue O (TB).

problem to solve is the instability of the composites, and dye molecules with small size are easy to leak out from the matrix [17]. Several approaches have been developed to incorporate organic dyes into a silica matrix, for instance, covalent coupling [18], reverse microemulsion [19], and electrostatic interaction (Stöber method) [20]. However, there is still no effective method to control the dispersion of the dye molecules, since they are quickly and spontaneously accumulated into the silica particles [21].

Herein, we reported a simple and modified Stöber method to synthesize $\mathrm{SiO}_{2}$-dyes composites by employing ultrasound irradiation during the hydrolysis and condensation steps of the silica source and dye addition. It was found that the as-synthesized composites were well dispersed into the silica particles with high stability during the photooxidation of 9,10-dimethylanthracene under visible light.

\section{Experimental}

2.1. Synthesis of $\mathrm{SiO}_{2}$-Dye Composites. Tetraethyl orthosilicate (TEOS), methylene blue (MB), safranin O (SF), toluidine blue (TB), neutral red (NR), and $\mathrm{SiO}_{2}$ nanopowder were purchased from Sigma-Aldrich and used without further purification. Ethanol (HPLC grade) was used as solvent; double distilled water and ammonium hydroxide $\left(\mathrm{NH}_{4} \mathrm{OH}\right)$ were also used during the synthesis. The molecular structure of the dyes can be seen in Figure 1 .

The composites were synthesized by using a modified Stöber method where the dye was incorporated since the formation of the $\mathrm{SiO}_{2}$ particles. TEOS was used as silica source and $\mathrm{NH}_{4} \mathrm{OH}$ as catalyst, and the composites silica-dye were obtained in one-step process. Briefly, it was prepared two solutions, one containing TEOS and ethanol (solution 1) and another with $\mathrm{NH}_{4} \mathrm{OH}$, water, and dye (solution 2). Solution 1 was poured drop by drop into solution 2, ultrasonic irradiation was applied during $10 \mathrm{~min}$, and the samples were aged for $12 \mathrm{~h}$ under constant agitation. The obtained powders were completely dried under vacuum at $318 \mathrm{~K}$. The molar ratios used in the preparation of the composites were $1 / 20 / 0.1 / 30$ for TEOS/water/ $\mathrm{NH}_{4} \mathrm{OH} /$ ethanol, and the nominal concentration of dye was $1 \times 10^{-5}$ or $1 \times 10^{-6}$ moles of dye/g of $\mathrm{SiO}_{2}$.

2.2. Characterization. The dynamic light scattering (DLS) measurements were determined by a Zetasizer Nano instrument (Malvern), and ethanol was used as dispersant. A Quanta 3D FEG (Fei) scanning electron microscope (SEM) was used; the dry samples were deposited onto a carbon tape before analysis. The $\mathrm{SiO}_{2}$ and $\mathrm{SiO}_{2}-\mathrm{MB}$ samples were analyzed in an Asap 2405 (Micromeritics) automated gas sorption system to obtain the nitrogen adsorption isotherms at $77.4 \mathrm{~K}$. The specific surface area was estimated with the Brunauer-Emmett-Teller (BET) model, and the pore size distribution was evaluated with the BJH model. All samples were outgassed at $373 \mathrm{~K}$ for $1 \mathrm{~h}$ prior to analysis. FTIR spectra in the region $4000-400 \mathrm{~cm}^{-1}$ were obtained with a Nexus 470 Spectrometer (Nicolet). The powders were mixed with $\mathrm{KBr}$ to form a pellet. UV-vis spectra were obtained on a Cintra 20 Spectrometer (GBC). The diffuse reflectance measurements were done using the lab sphere RSA-PE-20 accessory using $\mathrm{BaSO}_{4}$ as reference. In all cases the spectra were recorded in the $400-800 \mathrm{~nm}$ region.

2.3. Photocatalytic Evaluation. The photooxygenation of 9, 10-dimethylanthracene (DMA) was carried out in acetonitrile (HPLC grade) using a Newport solar simulator (Model 67005 ) equipped with a $150 \mathrm{~W}$ Xe lamp with a maximum emission around $460 \mathrm{~nm}$. It was used a $10 \mathrm{~mL}$ batch reactor for the evaluation of the $\mathrm{SiO}_{2}-\mathrm{MB}$ composites and an $80 \mathrm{~mL}$ batch reactor for the evaluation of the different $\mathrm{SiO}_{2}$-dye composites. The temperature was kept constant at $298 \mathrm{~K}$, and the incident light was filtered in order to cut out light below $400 \mathrm{~nm}$ and eliminate any photochemical reaction of DMA. The initial concentration of DMA was $16 \mathrm{mg} / \mathrm{L}$, and the composite loading was $1 \mathrm{~g} / \mathrm{L}$ or $0.5 \mathrm{~g} / \mathrm{L}$. The reaction samples were analyzed using HPLC (GBC model 1120) equipped with a UV detector $(\lambda=254 \mathrm{~nm})$, using a $70 / 30$ acetonitrile/water mobile phase $(1 \mathrm{~mL} / \mathrm{min}$ ) and a $15 \mathrm{~cm}$ column (Grace Prevail C18 $5 \mu$ ). The samples were also analyzed using a GC-MS 


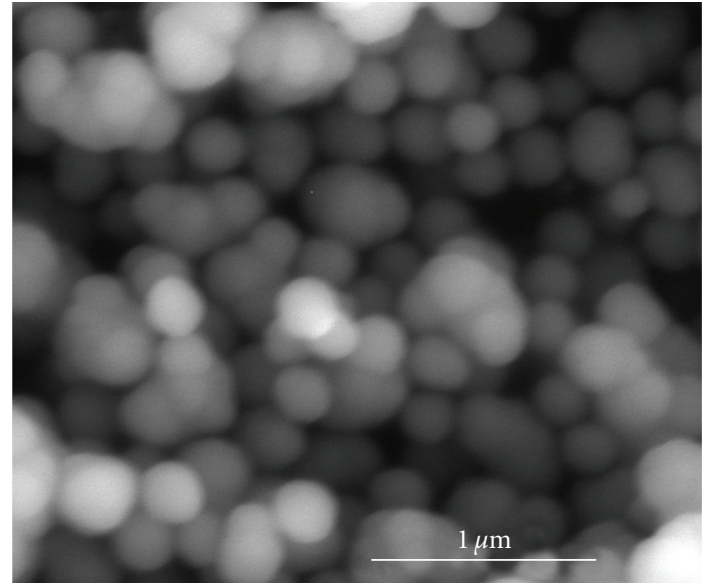

FIGURE 2: SEM image of $\mathrm{SiO}_{2}-\mathrm{MB}$ composite; $[\mathrm{MB}]=1 \times 10^{-5} \mathrm{~mol} /$ $\mathrm{g} \mathrm{SiO}_{2}$.

(Perkin-Elmer model TurboMass) using a $30 \mathrm{~m}$ capillary column (Alltech EC5).

\section{Results and Discussion}

3.1. Characterization Results of $\mathrm{SiO}_{2}-\mathrm{MB}$ Composites. The influence of the dye over the final particle size of the composite was determined by DLS. During the polymerization of TEOS through the formation of Si-O-Si bonds, the cationic part of $\mathrm{MB}$ molecule can interact with part of Si-O groups by electrostatic forces, increasing the stability of the hybrid system [17] and leading to an amount of the average size from $110 \mathrm{~nm}$ (as-prepared $\mathrm{SiO}_{2}$ ) to 141 and $185 \mathrm{~nm}$ by using $1 \times$ $10^{-6}$ and $1 \times 10^{5} \mathrm{~mol} / \mathrm{g} \mathrm{SiO}_{2} \mathrm{MB}$ concentration, respectively. The stability of the $\mathrm{SiO}_{2}-\mathrm{MB}$ composites was compared in a blank experiment, where a solution of $\mathrm{MB}$ in ethanol was mixed under stirring with the as-prepared $\mathrm{SiO}_{2}$ particles during $12 \mathrm{~h}$ and then dried under vacuum and $318 \mathrm{~K}$. The resulting material was rinsed with ethanol, and the $\mathrm{MB}$ was completely washed out, causing a total decolorization. However, the $\mathrm{SiO}_{2}-\mathrm{MB}$ composite prepared by the modified Stöber method never exhibited this behavior. The remaining ethanol used in the $\mathrm{SiO}_{2}-\mathrm{MB}$ composite washes was analyzed by UV-vis, and the characteristic absorption bands of $\mathrm{MB}$ were not observed, confirming the high stability of the composite.

A SEM image of the $\mathrm{SiO}_{2}-\mathrm{MB}$ composite shows the morphology of the spheres in Figure 2. It can be seen spheres ranging from 100 to $200 \mathrm{~nm}$ in size, in some cases well dispersed but also forming particle agglomerates. The morphology of the silica spheres (not shown here) was similar than that of the composite.

Figure 3 shows the nitrogen adsorption-desorption isotherm of the $\mathrm{SiO}_{2}$ and the $\mathrm{SiO}_{2}-\mathrm{MB}$ composite. The shape corresponds to a type IV isotherm according to the IUPAC classification [22]. The hysteresis loop (H3/H4 types) can be attributed to the presence of slit-shaped pores and open pores where no condensation was evident. According to these results, the pore size varies between 10 and $100 \mathrm{~nm}$ dia-meter (see Figure 3(a)). The corresponding surface
TABLE 1: Characteristic bands of different MB species [26].

\begin{tabular}{lcl}
\hline Species & Wavelength $(\mathrm{nm})$ & Assignment \\
\hline$\left(\mathrm{AM}^{+}\right)_{n}$ & $570-590$ & Trimer and higher aggregates \\
$\left(\mathrm{AM}^{+}\right)_{2}$ & $600-620$ & Dimer \\
$\mathrm{AM}^{+}$ & $650-675$ & Monomer \\
\hline
\end{tabular}

area was $91 \mathrm{~m}^{2} / \mathrm{g}$ for $\mathrm{SiO}_{2}$ and $23 \mathrm{~m}^{2} / \mathrm{g}$ for the $\mathrm{SiO}_{2}-\mathrm{MB}$ composite. Note that slight changes in the composite adsorption-desorption isotherm (Figure 3(b)) caused a dramatic decrease in specific surface area and a bimodal behavior of pore size distribution with an important amount of macropores. These results could be indicative that $\mathrm{MB}$ was incorporated, probably of irregular form, into the silica matrix modifying the shape and size of the pores.

FT-IR spectra of $\mathrm{MB}, \mathrm{SiO}_{2}$, and $\mathrm{SiO}_{2}-\mathrm{MB}$ composite $\left([\mathrm{MB}]=1 \times 10^{-5} \mathrm{~mol} / \mathrm{g} \mathrm{SiO}_{2}\right)$ are presented in Figure 4. The main absorption bands of MB at 1610, 1505, 1405, and $1355 \mathrm{~cm}^{-1}$ are assigned to the $=\mathrm{N}^{+}$cation, the heterocyclic skeleton, and to the $-\mathrm{CH}_{3}$ symmetric and asymmetric bending vibrations, respectively $[23,24]$ (Figure $4(\mathrm{a})$ ). The obtained spectrum of $\mathrm{SiO}_{2}$ (Figure 4(b)) reproduces the general features often reported for this compound [24, 25]. It is worth noting that bands at 1200, 1100, 800, and $460 \mathrm{~cm}^{-1}$ are attributed to $\mathrm{Si}-\mathrm{O}-\mathrm{Si}$ vibrations and band at $960 \mathrm{~cm}^{-1}$ corresponds to the $\mathrm{Si}-\mathrm{OH}$ vibration. The spectrum also presented a broad band located between 3750 and $3000 \mathrm{~cm}^{-1}$; this can be generated by the hydration of the solid (bands located at 3350 and 1630 [24]) or by the presence of $\mathrm{SiO}-\mathrm{H}$ vibrations [25]. The FT-IR spectrum of the $\mathrm{SiO}_{2}-\mathrm{MB}$ composite was quite similar than that of $\mathrm{SiO}_{2}$, which can be attributed to the low concentration of $\mathrm{MB}$ in the material, so that the main absorption bands of $\mathrm{MB}$ overlap with $\mathrm{SiO}_{2}$ bands (Figure $4(\mathrm{c})$ ). Note that a slight deformation of Si-O-Si bands at $1200 \mathrm{~cm}^{-1}$ and $3000-3750 \mathrm{~cm}^{-1}$ is detected in the composite which can be indicative of a bonding interaction between the organic dye and $\mathrm{SiO}_{2}$ [17].

The visible light absorption spectra of $\mathrm{SiO}_{2}-\mathrm{MB}$ composites and a mechanical mixture $\left(\mathrm{MB}+\right.$ commercial $\left.\mathrm{SiO}_{2}\right)$ are compared in Figure 5. In the mechanical mixture sample, a strong band appears at $670 \mathrm{~nm}$ and one shoulder at $610 \mathrm{~nm}$ (Figure 5(b)). The main band is associated to free molecules of $\mathrm{MB}$ (monomer, see Table 1), and the shoulder is assigned to the formation of the so-called $\mathrm{H}$-aggregates (dimers) $[26,27]$. In the diffuse reflectance spectrum of $\mathrm{SiO}_{2}-\mathrm{MB}$ composite $[\mathrm{MB}]=1 \times 10^{-6} \mathrm{~mol} / \mathrm{g} \mathrm{SiO}_{2}$ (Figure $5(\mathrm{a})$ ), a wide band appears with a maximum at around $610 \mathrm{~nm}$ indicating that the predominant species was $\mathrm{MB}$ dimers. If the concentration of $\mathrm{MB}$ is increased to $1 \times 10^{-5} \mathrm{~mol} / \mathrm{g} \mathrm{SiO}_{2}$, the maximum of absorption is shifted to around $590 \mathrm{~nm}$, which is interpreted as the formation of trimers and higher aggregates [26].

The visible light absorption spectra of the different $\mathrm{SiO}_{2}$ dye composites prepared by the modified Stöber method and the mechanical mixture preparations are shown in Figure 6. In general, each kind of preparations presented different optical properties. For instance, the composites prepared by the modified Stöber method showed a better 


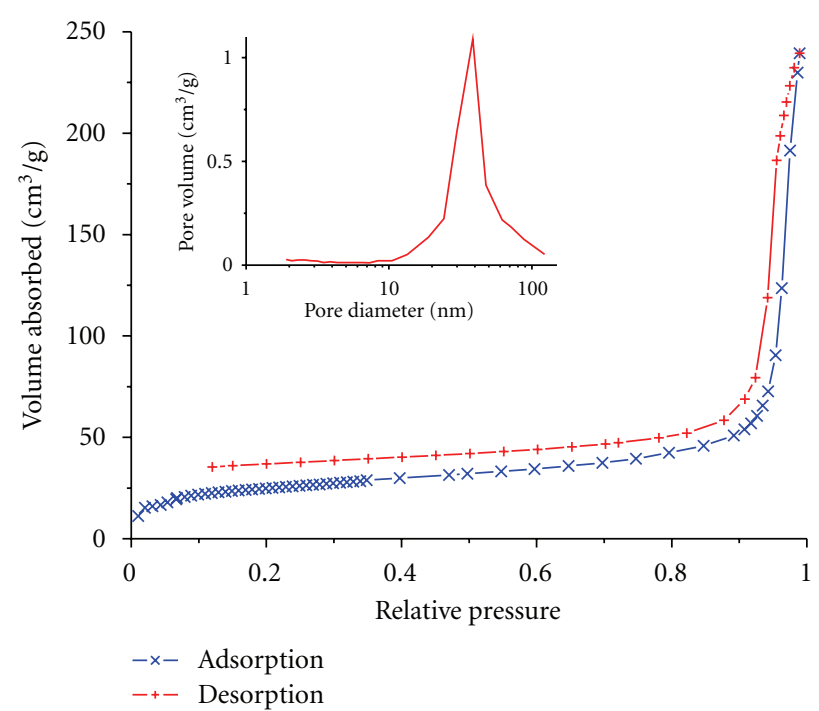

(a)

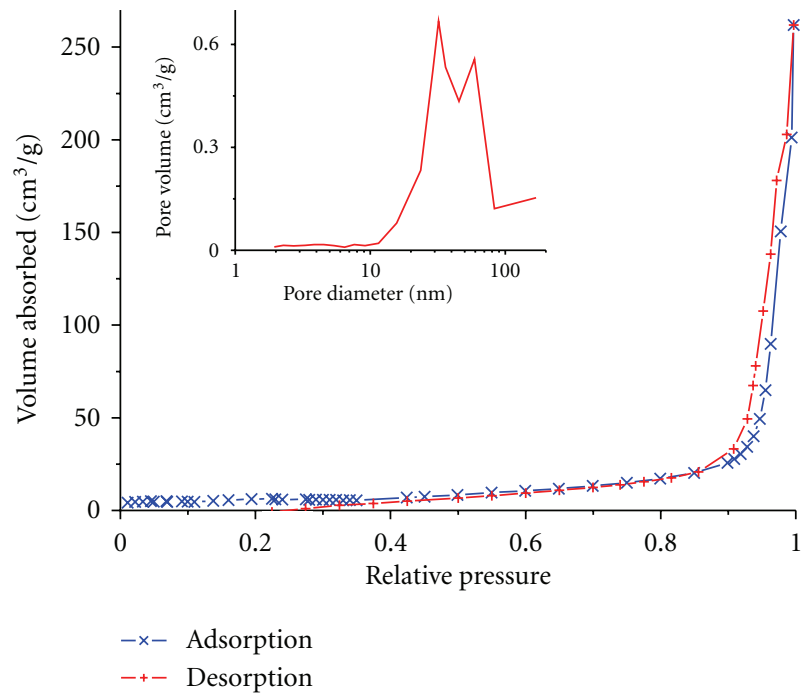

(b)

Figure 3: Nitrogen adsorption isotherms: (a) $\mathrm{SiO}_{2}$ material as-synthesized; (b) $\mathrm{SiO}_{2}-\mathrm{MB}$ composite; $[\mathrm{MB}]=1 \times 10^{-5} \mathrm{~mol} / \mathrm{g} \mathrm{SiO} 2$.

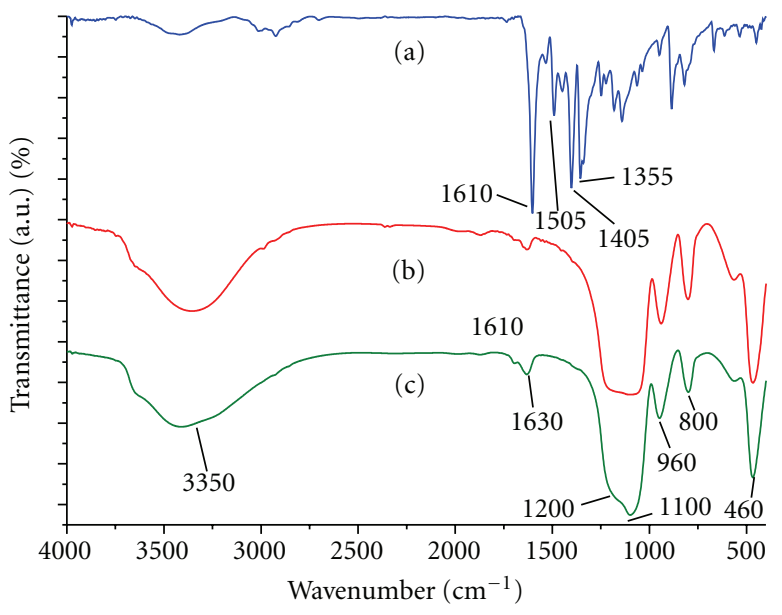

FIGURE 4: FT-IR spectra of (a) $\mathrm{MB}$ powder, (b) as-prepared $\mathrm{SiO}_{2}$, (c) $\mathrm{SiO}_{2}-\mathrm{MB},[\mathrm{MB}]=1 \times 10^{-5} \mathrm{~mol} / \mathrm{g} \mathrm{SiO}$.

band definition compared with the wide and irregular profile of the mechanical mixture bands. Note that a broad absorption band with a maximum at $490 \mathrm{~nm}$ was observed for commercial $\mathrm{SiO}_{2}+\mathrm{SF}$ (Figure 6(d)); in comparison with the $\mathrm{SiO}_{2}$-SF composite, a sharper absorption band appeared at $515 \mathrm{~nm}$ (Figure 6(a)). These results indicated that, in the first case, dye aggregates were the predominant species meanwhile; in the second case there were more monomeric dye species incorporated into the silica matrix $[28,29]$. The $\mathrm{SiO}_{2}-\mathrm{NR}$ composite and its mechanical mixture are shown in Figures 6(b) and 6(e). In the mechanical mixture, a broad absorption band from 400 to $650 \mathrm{~nm}$, with a maximum centered at $505 \mathrm{~nm}$, was previously assigned to the absorption of $\mathrm{H}$-aggregates (dimers) of the dye [29]. When the dye was incorporated since the formation of the $\mathrm{SiO}_{2}$ matrix, a solvatochromical shift in the absorption maximum

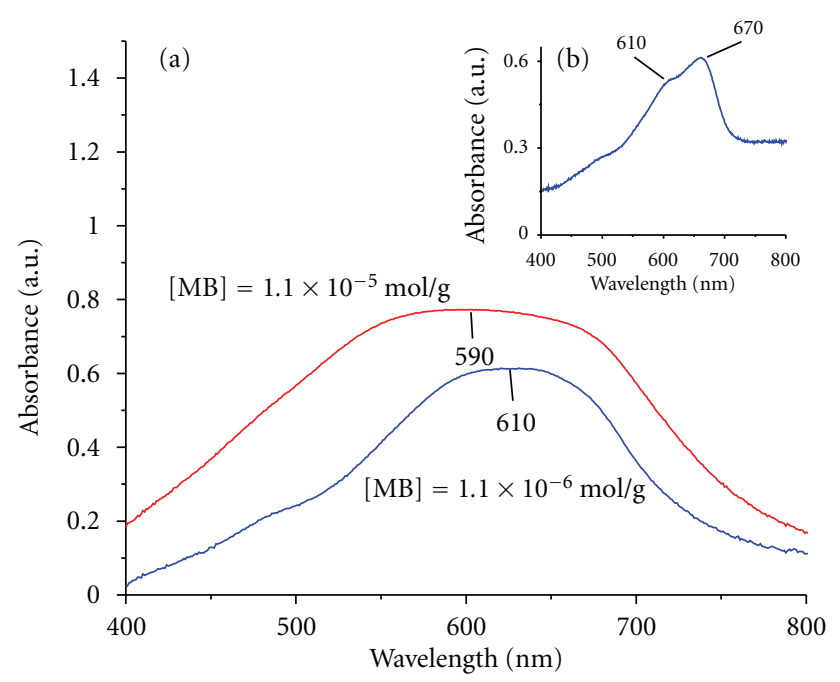

FIgURE 5: Visible light absorption spectra of (a) $\mathrm{SiO}_{2}$-dye nanocomposites and (b) mechanical mixture of $\mathrm{MB}+$ commercial $\mathrm{SiO}_{2}$ nanoparticles.

to $535 \mathrm{~nm}$ was absorbed, and a slight shoulder at $480 \mathrm{~nm}$ was also observed. The absorption band associated to the free molecules of NR is located around $535 \mathrm{~nm}$ [29-31], and the absorption associated with the uncharged form of NR is located around $450-460 \mathrm{~nm}$ in methanol [30, 32]. Hence, the predominant species in the $\mathrm{SiO}_{2}$-NR composite was the free molecules of the dye although the neutral form ( $\mathrm{pKa}=$ 6.8 [30]) could be present, due to basic media used in the preparation of the materials, causing the observed shoulder. In the case of $\mathrm{TB}$, the mechanical mixture with commercial $\mathrm{SiO}_{2}$ (Figure 6(f)), the absorption spectrum presented an illdefined band with a maximum centered at $585 \mathrm{~nm}$, produced by the presence of $\mathrm{TB}$ dimers $[33,34]$. When $\mathrm{TB}$ was 


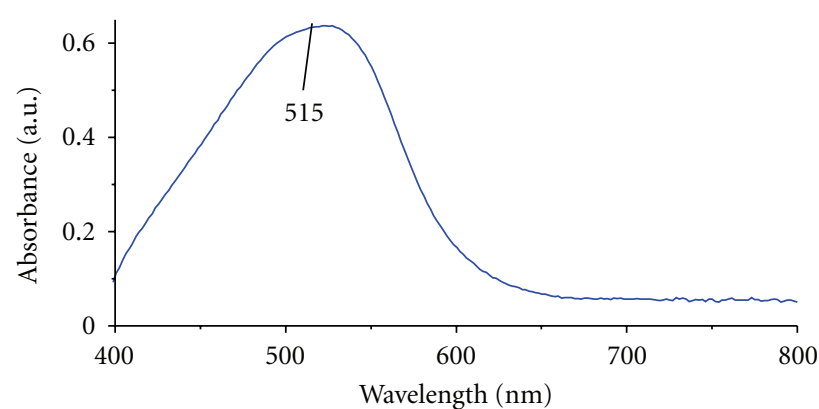

(a)

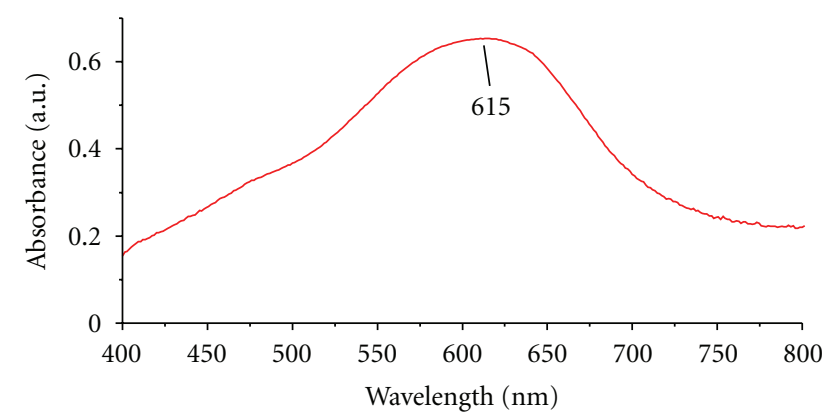

(c)

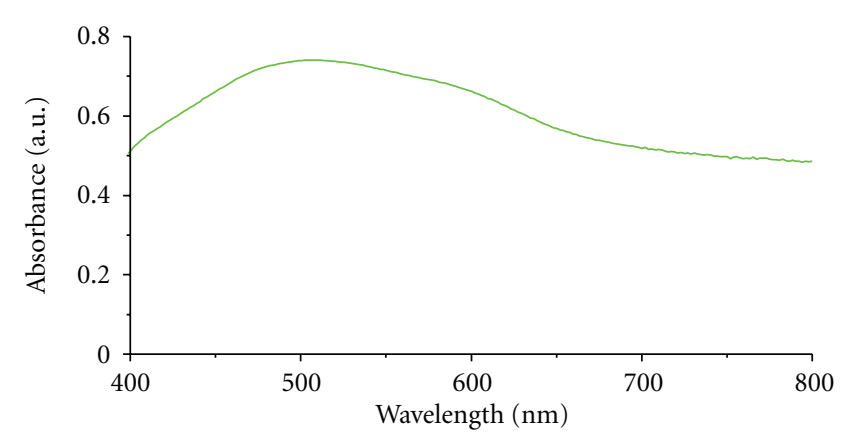

(e)

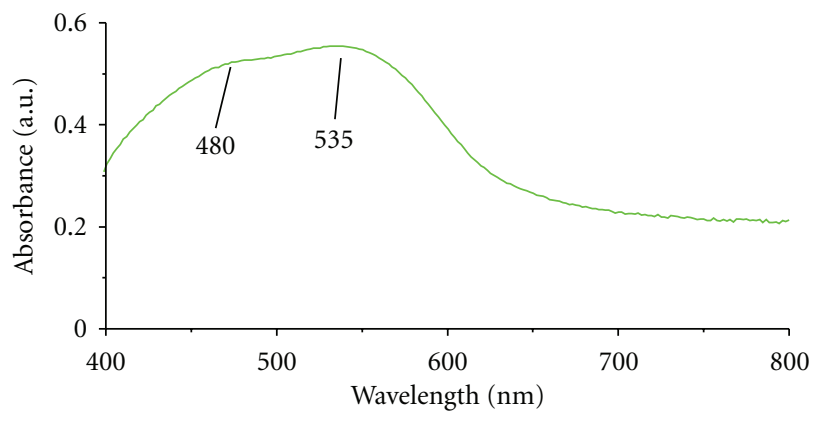

(b)

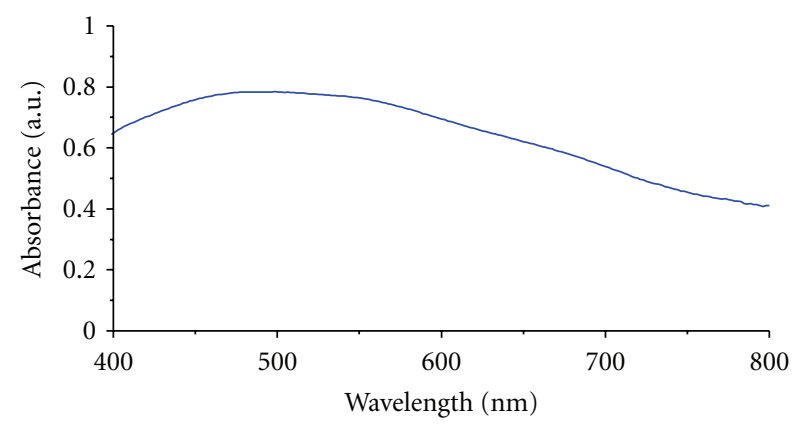

(d)

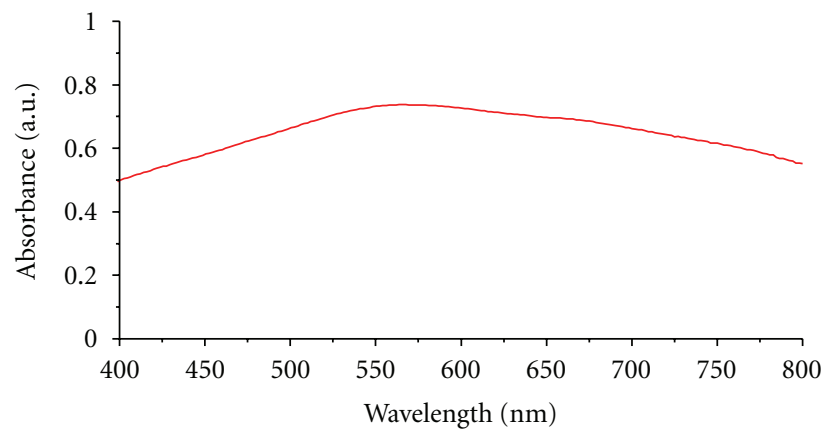

(f)

FIgURE 6: Visible light absorption spectra of $\mathrm{SiO}_{2}$-dye composites prepared by modified Stöber method: (a) SF, (b) NR, (c) TB, and prepared by mechanical mixture with commercial $\mathrm{SiO}_{2}$ : (d) SF, (e) NR, and (f) $\mathrm{TB}$.

incorporated into the $\mathrm{SiO}_{2}$ composites (Figure 6(c)), a welldefined band appeared in the absorption spectrum centered at $615 \mathrm{~nm}$ and a slight shoulder also appeared at $495 \mathrm{~nm}$. The absorption at $615 \mathrm{~nm}$ was produced by the presence of TB monomers in the composite.

3.2. Photosensitized Oxidation of DMA. In photosensitized reactions, the photosensitizer molecule absorbs the energy of a photon $(h v)$ of ultraviolet or visible radiation to become an excited singlet state which rapidly converts into an excited triple state. The lifetime of the triplet is longer (microseconds) than that of the singlet (nanoseconds) so that energy transfer from the triplet to dissolved oxygen molecule to form singlet oxygen $\left({ }^{1} \mathrm{O}_{2}\right)$ is possible. The amount of singlet oxygen generated by a photosensitizer is determined by the rate of absorption of photons, the triplet quantum yield, and the efficiency of the energy transfer process [35]. After singlet oxygen is generated, it either reacts with a substrate or losses its excitation energy as heat or light emission (phosphorescence). Scheme 1 shows a reaction scheme considering the visible light irradiation of a $\mathrm{SiO}_{2}$ dye composite in presence of oxygen, ethanol, and the main substrate DMA.

Scheme 1 (reaction scheme of the photosensitized oxidation of DMA on dye-doped silica composites).

$$
\begin{gathered}
\mathrm{SiO}_{2} \text {-dye }+h \nu \longrightarrow \mathrm{SiO}_{2} \text {-dye* } \quad I, \\
\mathrm{SiO}_{2} \text {-dye }{ }^{*}+{ }^{3} \mathrm{O}_{2} \longrightarrow \mathrm{SiO}_{2} \text {-dye }+{ }^{1} \mathrm{O}_{2} \quad k \\
\mathrm{DMA}+{ }^{1} \mathrm{O}_{2} \longrightarrow \text { DMA endoperoxide } \\
k_{c}, \\
{ }^{1} \mathrm{O}_{2} \longrightarrow{ }^{3} \mathrm{O}_{2} \quad k_{d},
\end{gathered}
$$

where $I$ is the light intensity, $k$ is a rate constant for the quenching of excited $\mathrm{SiO}_{2}$-dye by triplet oxygen to produce 


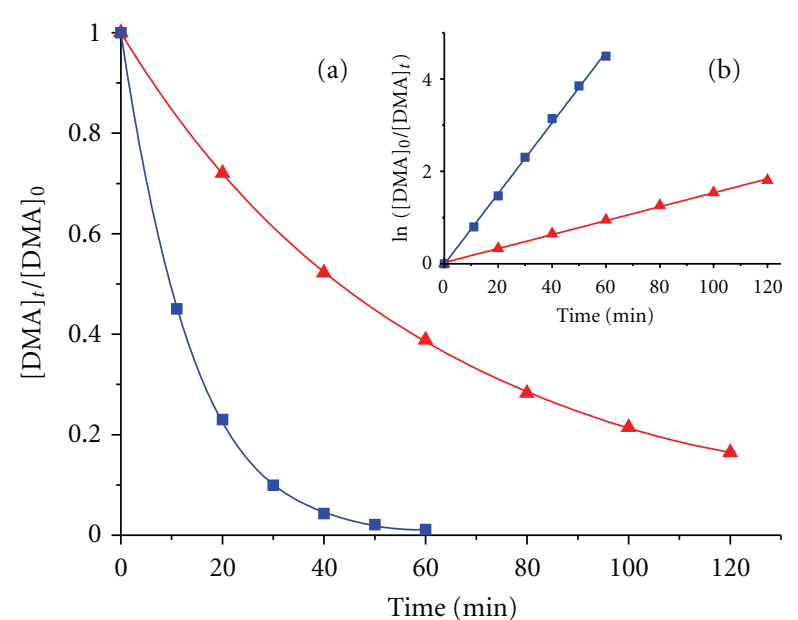

Figure 7: (a) Relative concentration of DMA versus time for $\mathrm{SiO}_{2}$ $\mathrm{MB}$ composites $(\boldsymbol{\Delta})[\mathrm{MB}]=1 \times 10^{-6} \mathrm{~mol} / \mathrm{g} \mathrm{SiO}_{2}(\boldsymbol{\square})[\mathrm{MB}]=1 \times$ $10^{-5} \mathrm{~mol} / \mathrm{g} \mathrm{SiO}_{2}$. (b) First-order kinetics data fitting.

singlet oxygen, $k_{c}$ is the rate constant of chemical quenching of singlet oxygen in presence of DMA; however, singlet oxygen also decays to the ground state by energy transfer to the solvent or with other species in solution with a rate constant $k_{d}$ [36]. A rough estimation of the rate constant in (3) can be obtained by considering that an excess of singlet oxygen is produced compared with the initial concentration of DMA and then a pseudofirst-order reaction is proposed (5)-(8):

$$
-\frac{d[\mathrm{DMA}]}{d t}=k_{c}[\mathrm{DMA}]\left[{ }^{1} \mathrm{O}_{2}\right]=k^{\prime}[\mathrm{DMA}]
$$

where

$$
\begin{gathered}
k^{\prime}=k_{c}\left[{ }^{1} \mathrm{O}_{2}\right]=\Phi I \frac{k c}{k d}, \\
{[\mathrm{DMA}]_{t}=[\mathrm{DMA}]_{0} e^{-k^{\prime} t},} \\
\ln \frac{[\mathrm{DMA}]_{0}}{[\mathrm{DMA}]_{t}}=k^{\prime} t
\end{gathered}
$$

The concentration profiles of DMA, during the photooxidation in presence of $\mathrm{SiO}_{2}-\mathrm{MB}$, are shown in Figure 7. Note that raw data fit well to a first-order reaction at two concentrations of $\mathrm{MB}$, with $k^{\prime}$ values of 0.0762 and $0.0154 \mathrm{~min}^{-1}$ for $1 \times 10^{-5}$ and $1 \times 10^{-6}$ moles of dye/g of $\mathrm{SiO}_{2}$, respectively.

As shown in Figure 8, the conversion of DMA followed a similar behavior (first order kinetics) with the different $\mathrm{SiO}_{2}$ dye composites. However, the rate constants had different values, and it is clearly seen that $\mathrm{SiO}_{2}-\mathrm{MB}$ is not a good photocatalyst for this reaction. Note that the $k^{\prime}$ values had the following increasing order depending of the dye used in the photooxidation of DMA (Figure 9): $\mathrm{SF}>\mathrm{TB}>\mathrm{NR}>$ $\mathrm{MB}$. These results revealed that the singlet oxygen generation depended on the type of composite used. Therefore, in the particular case of $\mathrm{SiO}_{2}-\mathrm{SF}$ composite instead of presenting almost the same absorption properties than $\mathrm{MB}$, it presented a rate constant one order of magnitude higher compared

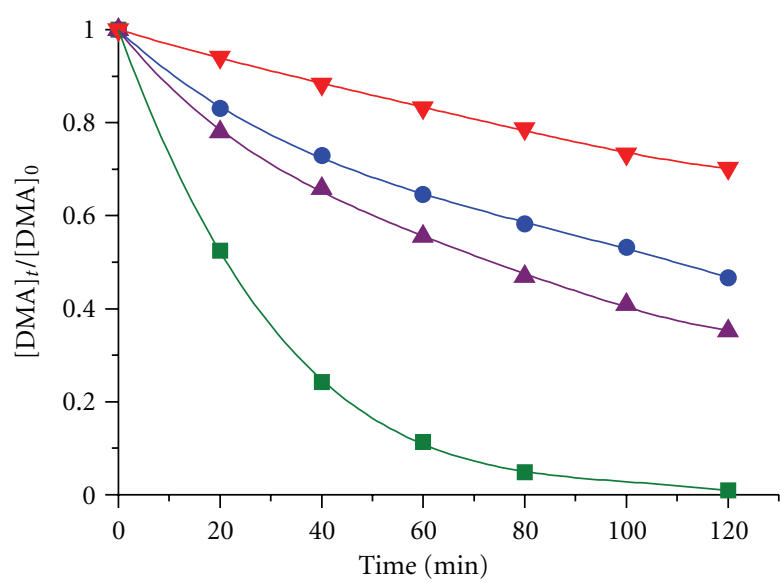

(a)

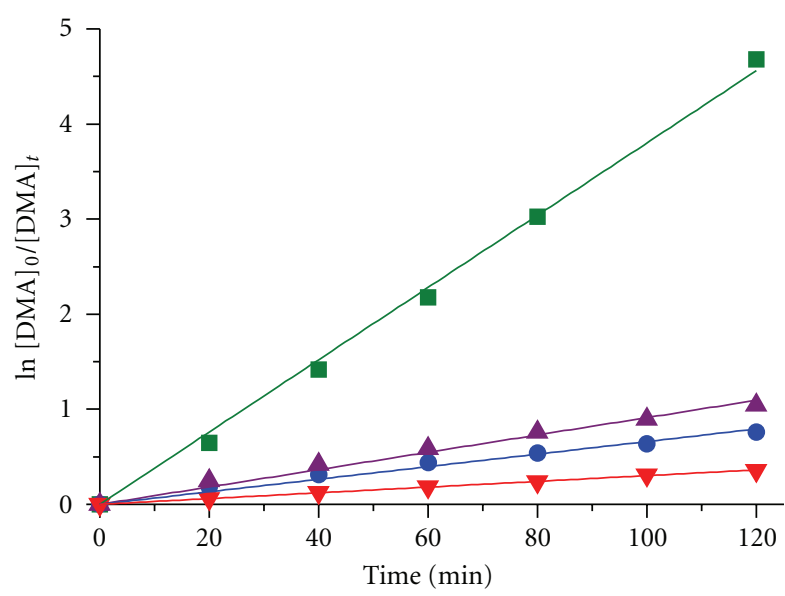

(b)

Figure 8: (a) Relative concentration of DMA versus time for different $\mathrm{SiO}_{2}$-composites: $(\boldsymbol{\nabla}) \mathrm{MB} ;(\bullet) \mathrm{NR} ;(\boldsymbol{\Delta}) \mathrm{TB}$; ( $\left.\mathbf{\square}\right) \mathrm{SF}$. (b) First-order kinetics data fitting.

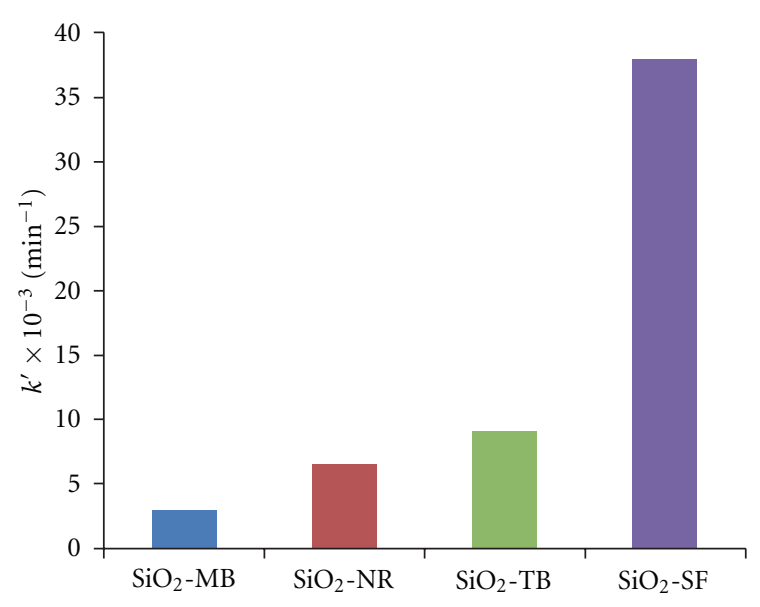

FIGURE 9: Rate constant values $\left(k^{\prime}\right)$ of the photooxidation of DMA with different $\mathrm{SiO}_{2}$-dye composites. 
<smiles></smiles>

FIGURE 10: Reaction path scheme of DMA endoperoxide formation as well as the unidentified V and VI products detected by HPLC and GC-MS (see Supplementary Information available online at doi: 10.1155/2012/987606).

with MB which should be interpreted as SF dye was homogenously dispersed on the silica matrix, as indicated by the UV-vis spectra results (Figures 5 and 6), where the $\mathrm{SiO}_{2}-\mathrm{SF}$ composite presented a sharper absorption band at $515 \mathrm{~nm}$, mainly as a consequence of the presence of monomeric species of SF in the material. Further studies are in progress in our lab in order to know in detail the properties and behavior of the $\mathrm{SiO}_{2}-\mathrm{SF}$ composites for fine chemical synthesis under visible light irradiation.

Finally, a reaction path scheme showing the formation of DMA endoperoxide and two unidentified byproducts ( $\mathrm{V}$ and VI), which were found with all series of dye-doped silica particles, is presented in Figure 10. As mentioned before, the main product was the DMA endoperoxide; however, the two unidentified byproducts were detected by HPLC and GCMS (supplementary information). As is well known, the endoperoxides are highly instable compounds so that easily decompose to other oxygenated compounds following a parallel path forming stable products as the unidentified $\mathrm{V}$ and VI byproducts.

\section{Conclusion}

In this study, a novel procedure (modified Stöber method) to obtain stable and active $\mathrm{SiO}_{2}$-dye composites for the photosensitized oxidation of 9,10-dimethylanthracene was developed. We found important differences in the mechanical mixtures and Stöber's method preparations of our four dye-doped silica composites than can be attributed to the higher electrostatic interaction and dispersion of the dye into the silica matrix by the second procedure. According to the results, all dyes had a larger affinity to the matrix and can be easily incorporated. These findings agreed with the photocatalytic behavior, and the $\mathrm{SiO}_{2}$-SF composite showed the most efficient delivery of ${ }^{1} \mathrm{O}_{2}$. Two byproducts were also detected during the photooxidation of DMA which are probably assigned to the decomposition of the endoperoxide.

\section{Acknowledgments}

This work was financially supported by the National Council of Science and Technology (Conacyt) Projects: 106891 and 153356. The authors also acknowledge the support of COFAA-IPN. E. Albiter thanks Conacyt for the scholarship support.

\section{References}

[1] C. Tanielian, C. Schweitzer, R. Seghrouchni, M. Esch, and R. Mechin, "Polyoxometalate sensitization in mechanistic studies of photochemical reactions: the decatungstate anion as a reference sensitizer for photoinduced free radical oxygenations of organic compounds," Photochemical and Photobiological Sciences, vol. 2, no. 3, pp. 297-305, 2003.

[2] G. Schenck, "Photosensitization," Industrial \& Engineering Chemistry, vol. 55, no. 6, pp. 40-43, 1963.

[3] C. S. Foote, "Mechanisms of photosensitized oxidation," Science, vol. 162, no. 3857, pp. 963-970, 1968.

[4] C. S. Foote, "Type I and type II mechanisms of photodynamic action," in Light-Activated Pesticides, vol. 339, pp. 22-38, American Chemical Society, 1987.

[5] A. Greer, "Christopher Foote's discovery of the role of singlet oxygen $\left[{ }^{1} \mathrm{O}_{2}\right]$ in photosensitized oxidation reactions," Accounts of Chemical Research, vol. 39, no. 11, pp. 797-804, 2006.

[6] E. L. Clennan and A. Pace, "Advances in singlet oxygen chemistry," Tetrahedron, vol. 61, no. 28, pp. 6665-6691, 2005.

[7] M. C. DeRosa and R. J. Crutchley, "Photosensitized singlet oxygen and its applications," Coordination Chemistry Reviews, vol. 233-234, pp. 351-371, 2002.

[8] J. Wahlen, D. E. De Vos, P. A. Jacobs, and P. L. Alsters, "Solid materials as sources for synthetically useful singlet oxygen," Advanced Synthesis and Catalysis, vol. 346, no. 2-3, pp. 152164, 2004.

[9] S. Wang, R. Gao, F. Zhou, and M. Selke, "Nanomaterials and singlet oxygen photosensitizers: potential applications in photodynamic therapy," Journal of Materials Chemistry, vol. 14, no. 4, pp. 487-493, 2004.

[10] A. E. O’Connor, W. M. Gallagher, and A. T. Byrne, "Porphyrin and nonporphyrin photosensitizers in oncology: preclinical and clinical advances in photodynamic therapy," Photochemistry and Photobiology, vol. 85, no. 5, pp. 1053-1074, 2009.

[11] P. Couleaud, V. Morosini, C. Frochot, S. Richeter, L. Raehm, and J. O. Durand, "Silica-based nanoparticles for photodynamic therapy applications," Nanoscale, vol. 2, no. 7, pp. 10831095, 2010.

[12] T. Montagnon, M. Tofi, and G. Vassilikogiannakis, "Using singlet oxygen to synthesize polyoxygenated natural products from furans," Accounts of Chemical Research, vol. 41, no. 8, pp. 1001-1011, 2008.

[13] D. Gryglik, J. S. Miller, and S. Ledakowicz, "Solar energy utilization in degradation of 2-chlorophenol by immobilized photosensitizers," Solar Energy, vol. 77, no. 5, pp. 615-623, 2004.

[14] L. Villén, F. Manjón, D. García-Fresnadillo, and G. Orellana, "Solar water disinfection by photocatalytic singlet oxygen production in heterogeneous medium," Applied Catalysis B, vol. 69, no. 1-2, pp. 1-9, 2006.

[15] F. M. P. R. Van Laar, F. Holsteyns, I. F. J. Vankelecom, S. Smeets, W. Dehaen, and P. A. Jacobs, "Singlet oxygen generation using PDMS occluded dyes," Journal of Photochemistry and Photobiology A, vol. 144, no. 2-3, pp. 141-151, 2001.

[16] A. S. de Dios and M. E. Díaz-García, "Multifunctional nanoparticles: analytical prospects," Analytica Chimica Acta, vol. 666, no. 1-2, pp. 1-22, 2010.

[17] H. Yao, J. M. Hong, N. Li, S. Xu, and J. J. Zhu, "Homogenous thionine- $\mathrm{SiO}_{2}$ nanocomposite spheres: sonochemical preparation, characterization, and application in $\mathrm{H}_{2} \mathrm{O}_{2}$ biosensor," Journal of Nanoscience and Nanotechnology, vol. 9, no. 4, pp. 2421-2425, 2009. 
[18] A. van Blaaderen and A. Vrij, "Synthesis and characterization of colloidal dispersions of fluorescent, monodisperse silica spheres," Langmuir, vol. 8, no. 12, pp. 2921-2931, 1992.

[19] F. J. Arriagada and K. Osseo-Asare, "Synthesis of nanosize silica in aerosol OT reverse microemulsions," Journal of Colloid And Interface Science, vol. 170, no. 1, pp. 8-17, 1995.

[20] W. Stöber, A. Fink, and E. Bohn, "Controlled growth of monodisperse silica spheres in the micron size range," Journal of Colloid And Interface Science, vol. 26, no. 1, pp. 62-69, 1968.

[21] D. Zhang, Z. Wu, J. Xu, J. Liang, J. Li, and W. Yang, “Tuning the emission properties of $\mathrm{Ru}(\mathrm{phen})_{3}^{2+}$ doped silica nanoparticles by changing the addition time of the dye during the stöber process," Langmuir, vol. 26, no. 9, pp. 6657-6662, 2010.

[22] K. S. W. Sing, D. H. Everett, R. A. W. Haul et al., "Reporting physorption data for gas/solid systems," Pure and Applied Chemistry, vol. 57, no. 4, p. 16, 1985.

[23] O. V. Ovchinnikov, S. V. Chernykh, M. S. Smirnov et al., "Analysis of interaction between the organic dye methylene blue and the surface of $\mathrm{AgCl}(\mathrm{I})$ microcrystals," Journal of Applied Spectroscopy, vol. 74, no. 6, pp. 809-816, 2007.

[24] K. Nakanishi and P. H. Solomon, Infrared Absorption Spectroscopy, Holden-Day, San Francisco, Calif, USA, 1977.

[25] G. D. Chukin and V. I. Malevich, "Infrared spectra of silica," Journal of Applied Spectroscopy, vol. 26, no. 2, pp. 223-229, 1977.

[26] J. J. Beaudoin, B. Patarachao, L. Raki, and R. Alizadeh, "Adsorption of methylene blue as a descriptor of C-S-H nanostructure," Cement and Concrete Composites, vol. 33, no. 2, pp. 246-250, 2011.

[27] D. Madhavan and K. Pitchumani, "Reactions in clay media: photooxidation of sulfides by clay-bound methylene blue," Tetrahedron, vol. 57, no. 39, pp. 8391-8394, 2001.

[28] M. L. Gómez, C. M. Previtali, and H. A. Montejano, "Photophysical properties of safranine O in protic solvents," Spectrochimica Acta A, vol. 60, no. 11, pp. 2433-2439, 2004.

[29] L. Antonov, G. Gergov, V. Petrov, M. Kubista, and J. Nygren, "UV-Vis spectroscopic and chemometric study on the aggregation of ionic dyes in water," Talanta, vol. 49, no. 1, pp. 99106, 1999.

[30] M. K. Singh, H. Pal, A. C. Bhasikuttan, and A. V. Sapre, "Dual solvatochromism of neutral red," Photochemistry and Photobiology, vol. 68, no. 1, pp. 32-38, 1998.

[31] F. Gessner, C. C. Schmitt, and M. G. Neumann, "Time-dependent spectrophotometric study of the interaction of basic dyes with clays. 1 . Methylene blue and neutral red on montmorrillonite and hectorite," Langmuir, vol. 10, no. 10, pp. 3749-3753, 1994.

[32] J. Mohanty, A. C. Bhasikuttan, W. M. Nail, and H. Pal, "Hostguest complexation of neutral red with macrocyclic host molecules: contrasting $\mathrm{pK}_{a}$ shifts and binding affinities for cucurbit[7] uril and $\beta$-cyclodextrin," Journal of Physical Chemistry B, vol. 110, no. 10, pp. 5132-5138, 2006.

[33] M. Ilanchelian, C. Retna Raj, and R. Ramaraj, "Spectral studies on the Cyclodextrin inclusion complexes of toluidine blue $\mathrm{O}$ and Meldola's blue in aqueous solution," Journal of Inclusion Phenomena, vol. 36, no. 1, pp. 9-20, 2000.

[34] J. Jebaramya, M. Ilanchelian, and S. Prabahar, "Spectral studies of toluidine blue o in the presence of sodium dodecyl sulfate," Digest Journal of Nanomaterials and Biostructures, vol. 4, no. 4, pp. 789-797, 2009.

[35] C. S. Foote, "Photosensitized oxygenations and the role of singlet oxygen," Accounts of Chemical Research, vol. 1, no. 4, pp. 104-110, 1968.
[36] W. Tang, H. Xu, R. Kopelman, and M. A. Philbert, "Photodynamic characterization and in vitro application of methylene blue-containing nanoparticle platforms," Photochemistry and Photobiology, vol. 81, no. 2, pp. 242-249, 2005. 


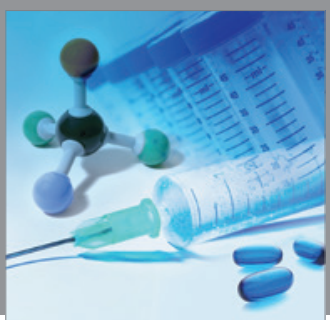

International Journal of

Medicinal Chemistry

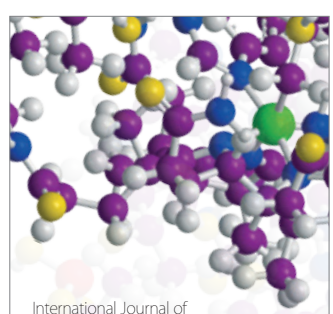

Carbohydrate Chemistry

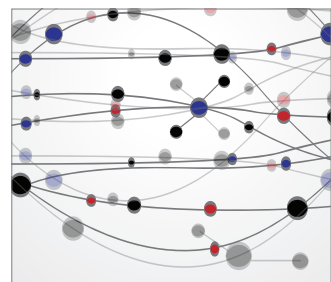

The Scientific World Journal
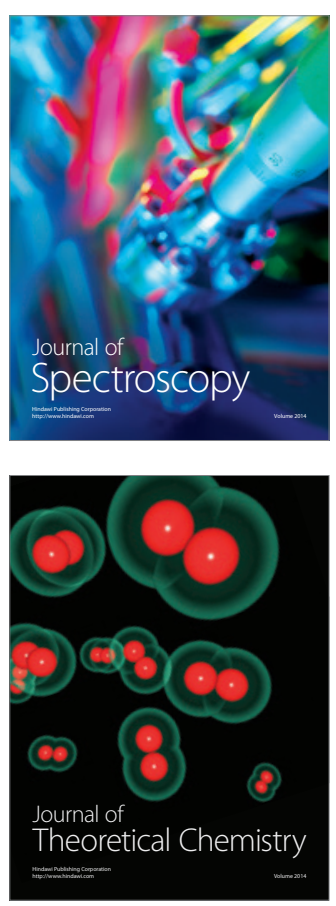
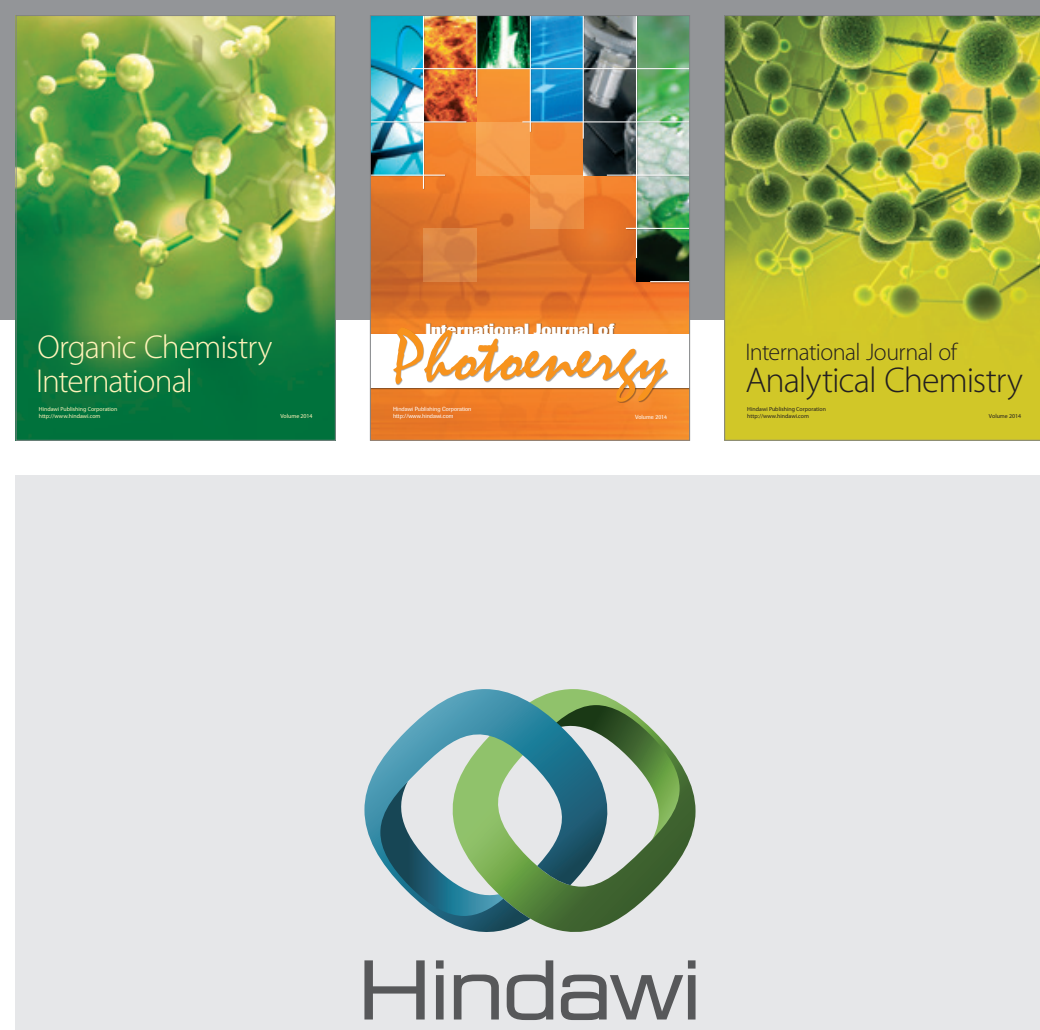

Submit your manuscripts at

http://www.hindawi.com
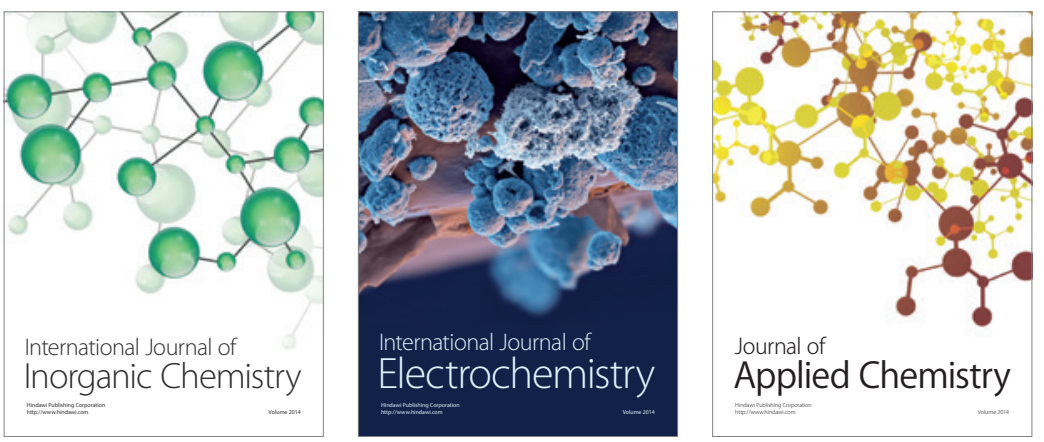

Journal of

Applied Chemistry
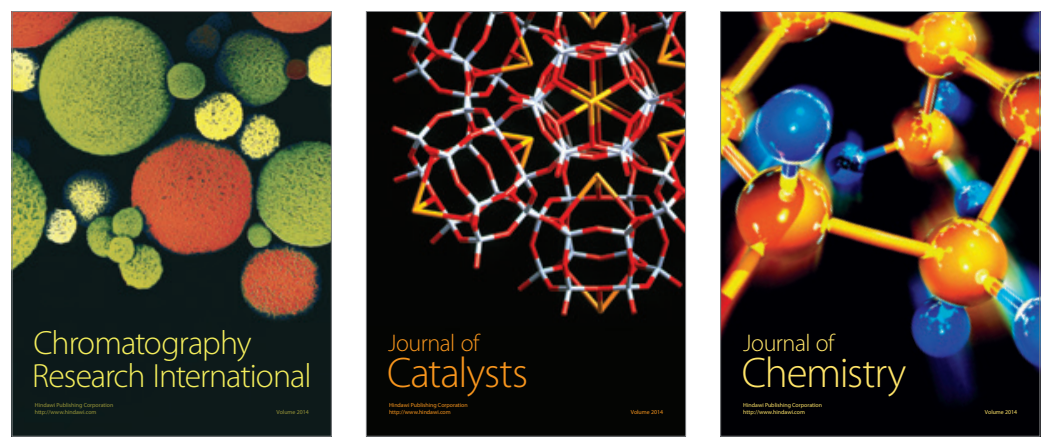
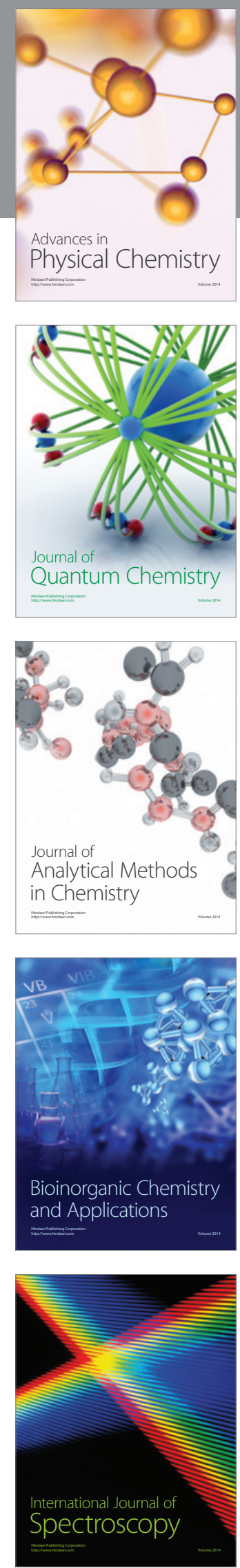